\title{
DENSIDADES DE SIEMBRA, ARREGLOS ESPACIALES Y FERTILIZACIÓN EN PEJIBAYE (Bactris gasipaes cv 'DIAMANTES-10') PARA PALMITO ${ }^{1}$
}

\author{
Francisco Paulo Chaimsohn ${ }^{2}$, Jorge Mora-Urpi ${ }^{3}$, Enrique Villalobos-Rodríguez ${ }^{4}$
}

\section{RESUMEN}

Densidades de siembra, arreglos espaciales y fertilización en pejibaye (Bactris gasipaes cv Diamantes-10) para palmito. La investigación se llevó a cabo en la Estación Experimental Los Diamantes (Guápiles, Costa Rica), el 3 de octubre del 2003, cuyo objetivo fue la evaluación del efecto de diferentes densidades de siembra $(3.333,5.000 \mathrm{y}$ 6.666 plantas/ha), arreglos espaciales, y diversos métodos de fertilización (química, orgánica), sobre el crecimiento de las plantas de pejibaye para producción de palmito. Se consideraron las variables diámetro y altura del tallo primario y el número de hojas y rebrotes como indicadores de producción. El período de evolución abarcó sólo los primeros 25 meses de crecimiento en el campo. El número de hojas, la altura y el diámetro del tallo no mostraron diferencias de respuesta relevantes. Sólo el número de rebrotes disminuyó al aumentar la densidad de la población, cuando se midió a los 15 meses de edad. El efecto de la fertilización se hizo evidente después de la primera cosecha, realizada a los 20 meses, debido al aumento de la competencia entre plantas, ahora más desarrolladas. Fue entonces cuando la fertilización química indujo la producción de un mayor número y vigor de los rebrotes. Sin embargo, las prácticas evaluadas 25 meses después de la siembra, no habían influido hasta ese momento en el número de palmitos cosechados, ni tampoco había afectado las características físicas de los sectores foliar y caulinar del palmito.

Palabras clave: Fertilización orgánica, fenología, rendimiento, calidad.

\begin{abstract}
Plant populations, arrangement and fertilizer type in peach palm (Bactris gasipaes cv 'Diamantes-10') for palm heart production. The field trial was carried out at Experimental Station "Los Diamantes" (Guápiles, Costa Rica) from October 2003 to November 2005. The objective was to evaluate the effect of different plant densities (3 333, 5000 and 6666 plants/ha), diverse methods of fertilization (chemical, organic and chemical-organic) and two spatial disposition of plants in the field (rectangular and triangular), on the growth of the peach palm for palm heart. The variables considered were stem diameter and height as well as leaf and sprout numbers, as plant production indicators. The evaluation period covered only the first 25 months of growth after planting in the open field. The number of leaves, plant height and stem diameter did not show relevant differences of response to the treatments. After 15 months, only the number of sprouts diminished as the population density increased. The effect of the fertilization became evident after the first harvest, 20 months after fields planting, due to the increased competition among more developed older plants. At this time chemical fertilizers caused an increased number and vigour of the sprouts. But the agronomical practices, evaluated 25 months after planting, did not produce any influence on the number of palm hearts harvested or on the physical characteristics of the foliar and caulinar sections of the heart of palm.
\end{abstract}

Key words: Organic fertilization, phenology, yield, quality.

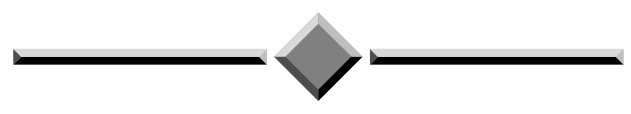

Recibido: 10 de enero, 2007. Aceptado: 29 de agosto, 2007. *Parte de la tesis de doctorado en Sistemas de Producción Agrícola Tropical Sostenible, Facultad de Ciencias Agroalimentarias, Universidad de Costa Rica.

2 Instituto Agronômico do Paraná (IAPAR). Becado de Doctorado en el exterior por el CNPq - Brasil. Correo electrónico: fpchaimsohn@ yahoo.com.br.

3 Escuela de Biología, Universidad de Costa Rica, San José, Costa Rica. Correo electrónico: pejibaye@cariari.ucr.ac.cr.

4 Centro de Investigación en Granos y Semillas (CIGRAS), Universidad de Costa Rica, San José, Costa Rica. Correo electrónico: villalobos. enrique@gmail.com. 


\section{INTRODUCCIÓN}

El área de cultivo del pejibaye para la producción de palmito ha experimentado un marcado crecimiento en Brasil, Ecuador, Guyana, Venezuela, Bolivia, Colombia, Perú y en algunos países asiáticos como Filipinas y Tailandia (Pereira 2002). Aunque el área cultivada en Costa Rica ha mostrado una disminución en los últimos años, el palmito sigue siendo un producto agrícola importante en la economía costarricense, cuyo valor total de las exportaciones en 2005 superó los US\$ 19,6 millones (PROCOMER 2006).

Actualmente, la producción de palmito se destina, casi en su totalidad, a suplir la demanda de materia prima para la industria en conserva. Sin embargo, se considera que hay un mercado potencial importante para el palmito al natural y/o de procesamiento mínimo (Clement et al. 1996; Chaimsohn 2000). La producción de este tipo de palmito es más apropiada para los pequeños agricultores, ya que no genera dependencia de una industria para el procesamiento y comercialización de su cosecha. Por otra parte, permite la apertura de mercados alternativos en el ámbito local, nacional e internacional, adicionalmente al mercado existente del producto en conserva (Chaimsohn 2000).

El palmito para consumo al natural requiere tecnología de producción diferenciada en comparación con el palmito destinado a la industria, dado que su procesamiento y comercialización son distintos, principalmente para mercados que exigen una alta calidad. La producción de palmito orgánico podría agregar valor al producto y facilitar la comercialización del palmito en nichos de mercado con preferencia por este tipo de productos.

Entre los factores ambientales que influyen en el rendimiento y en la calidad del palmito, además de la variedad, la densidad de siembra y la fertilización son de los más importantes porque definen las características físicas, químicas y sensoriales del producto. La demanda de un palmito de mayor diámetro, como lo exige el mercado de palmito al natural, puede obligar a sembrar a una menor densidad de población (Mora Urpí et al. 1999).

Nascimento et al. (2005) señalan que el pejibaye cultivado para la producción de palmito responde positivamente a la aplicación de enmienda orgánica, pero la información sobre fuentes y cantidades de este tipo de enmiendas es aún escasa.

La presente investigación tuvo como objetivo evaluar el crecimiento de las plantas y la producción inicial de palmito para consumo al natural en función de la densidad, arreglo espacial de la plantación y fertilizaciones química, orgánica y mixta.

\section{MATERIALES Y MÉTODOS}

El experimento se instaló en un área de la Estación Experimental Los Diamantes, propiedad del Ministerio de Agricultura y Ganadería, ubicada en Guápiles, cantón Pococí, provincia de Limón, Costa Rica; coordenadas $10^{\circ} 22^{\prime}$ latitud norte y $83^{\circ} 76^{\prime}$ longitud oeste, a una altitud de $249 \mathrm{msnm}$. De octubre del 2003 a octubre del 2005 la temperatura promedio fue de $24,5^{\circ} \mathrm{C}$ y la precipitación anual de 4.179,6 mm. (Figura 1).

El suelo se clasificó como un Andic Oxyaquic Dystrudepts (USDA y NRCS 2006), y presenta textura franco arenosa. Se realizó un análisis del suelo, para tener una caracterización de las propiedades químicas del mismo, el cual se muestra en el Cuadro 1.

El terreno se preparó con dos pasadas de arado y dos de rastra en enero del 2003. El manejo de malezas, especialmente Paspalum fasciculatum, se hizo con glifosato.

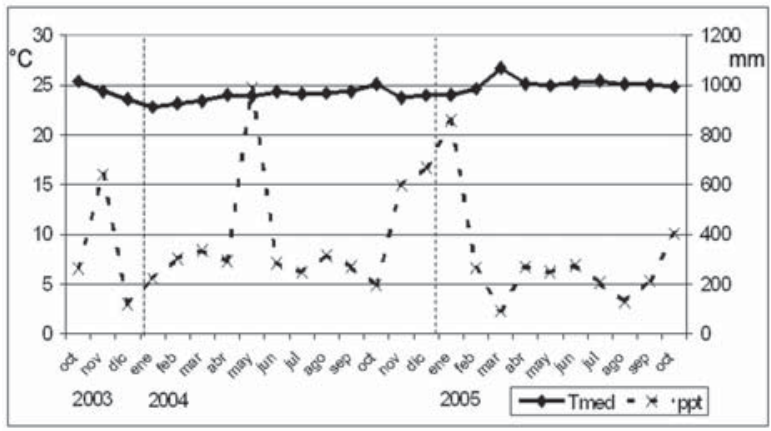

Figura 1. Temperatura promedio y precipitación mensuales en el período de octubre de 2003 a octubre de 2005. Estación Experimental Los Diamantes, Limón, Costa Rica. 
Cuadro 1. Análisis químico del suelo. Estación Experimental Los Diamantes, Limón, Costa Rica. 2003.

\begin{tabular}{|c|c|c|c|c|c|c|c|c|c|c|}
\hline \multirow{2}{*}{$\begin{array}{l}\mathbf{p H} \\
\mathrm{H}_{2} \mathrm{O}\end{array}$} & \multicolumn{4}{|c|}{$\operatorname{Cmol}(+) / 1$} & \multirow[b]{2}{*}{ CICE } & \multicolumn{5}{|c|}{$\mathrm{mg} / \mathrm{l}$} \\
\hline & $\mathrm{Ca}$ & $\mathrm{Mg}$ & $\mathrm{K}$ & $\mathrm{Al}$ & & $\mathrm{P}$ & $\mathrm{Cu}$ & $\mathrm{Fe}$ & $\mathrm{Mn}$ & $\mathrm{Zn}$ \\
\hline 4,9 & 4,0 & 1,7 & 0,5 & 0,4 & 6,6 & 4,3 & 9,8 & 333 & 17,3 & 3,0 \\
\hline
\end{tabular}

La siembra en el campo se hizo el 2 y 3 de octubre del 2003. El almácigo utilizado, del cultivar sin espinas 'Diamantes-10', tenía tres meses de germinado y dos pares de hojas formadas. Se resembraron las plantas perdidas, en forma sistemática, hasta febrero del 2004.

Las malezas se combatieron con chapeas y con aplicaciones de herbicidas [glifosato + (2,4 D)], conforme el tipo e infestación de las malezas.

En noviembre del 2003, 40 días después de la siembra (dds) y en el período de enero a abril del 2004 (132 a 201 dds) se aplicó fosfato diamónico (30 g/planta) mensualmente para promover el desarrollo de las raíces y homogeneizar la plantación experimental.

La hormiga Solenopsis geminata se combatió con la aplicación localizada de insecticida (malathion) a partir de mayo del 2004 debido al alto grado de infestación.

Los tratamientos evaluados fueron la combinación de densidades y arreglos de población con tipos de fertilización. Se utilizaron tres densidades de siembra: $3.333,5.000$ y 6.666 plantas/ha; dos arreglos o disposiciones de las plantas: rectangular y triangular ("pata de gallo"); y tres tipos de fertilización: a) Testigo: consistió en la aplicación de fertilizante químico, de acuerdo con la recomendación que se da para la Zona Atlántica de Costa Rica (Molina 2000), en función del análisis del suelo; b) Abono orgánico (compost): se ajustaron las cantidades de la enmienda orgánica a la concentración de nitrógeno de los abonos químicos utilizados; c) Abono mixto: este tratamiento consistió en aplicar una parte del fertilizante en forma orgánica y una parte en forma química, de acuerdo con el análisis del suelo y del compost (Cuadros 2 y 3). Es importante señalar que el cálculo del fertilizante a aplicar se hizo por planta y no por área. De esta forma, las parcelas de menor densidad recibieron la mitad del fertilizante por área que aquellas de mayor densidad. Por ejemplo, la enmienda orgánica se aplicó en una cantidad de $8,48 \mathrm{~kg} /$ planta/año, lo que correspondió a un total de 28, 42 y $57 \mathrm{t} / \mathrm{ha} / \mathrm{año}$, respectivamente para las parcelas con densidades de 3.333, 5.000 y 6.666 plantas/ha (Cuadro 4).

Se usó la cámara de presión de Scholander para monitorear el estado hídrico de las plantas antes de la salida del sol, con resultados negativos sobre su posible exposición al estrés hídrico en aquellas ocasiones cuando la lluvia disminuyó esporádicamente por períodos cortos, no mayores a cinco días. Se adaptó la metodología utilizada por Villalobos et al. 1992 para las mediciones del potencial hídrico palma aceitera.

Se utilizó un arreglo factorial de parcelas divididas, en un diseño de bloques completos al azar, con cuatro repeticiones, donde la parcela principal fue la densidad combinada con el arreglo de siembra y las subparcelas los tratamientos de fertilización. Las parcelas medían alrededor de $200 \mathrm{~m}^{2}$ y el área experimental total fue de aproximadamente $4.800 \mathrm{~m}^{2}$.

Las evaluaciones dendrométricas y fenológicas trimestrales se iniciaron a los 195 dds, en abril de 2004. El día previo a cada cosecha se evaluaron el número de hojas vivas y el número de estípites, de conformidad con las recomendaciones de Clement y Bovi (1999).

La cantidad y desarrollo de los rebrotes se evaluó en mayo y agosto de 2005: se determinó el número de rebrotes catalogándolos en dos grupos (mayor y menor que $3 \mathrm{~cm}$ de diámetro) por planta y el número de hojas, la altura y el diámetro basal del rebrote más desarrollado en cada planta. Aproximadamente 16 meses después de la siembra (mds) (segunda y tercera semanas de febrero de 2005) se efectuó la cosecha del palmito de las plantas útiles, cuyo diámetro del tallo era igual o superior a los $10 \mathrm{~cm}$. La cosecha de los tallos primarios, no 
Cuadro 2. Fertilizantes químicos y enmiendas orgánicas utilizados en los tratamientos experimentales aplicados a diferentes días después de la siembra. Estación Experimental Los Diamantes, Limón, Costa Rica. 2003-2005.

\begin{tabular}{llccr}
\hline \multirow{2}{*}{ dds } & \multicolumn{1}{c}{ Material $^{\dagger}$} & \multicolumn{3}{c}{ Fertilización - g/planta } \\
\cline { 3 - 5 } & & químico & orgánico & quim.-org. \\
\hline $\mathbf{6 8}$ & fosfato diamónico - DAP & 40 & - & 20 \\
& boñiga & - & 2000 & 1000 \\
$\mathbf{2 3 6}$ & 18-5-15-6-0,7 & 30 & - & 15 \\
& lombri compost (1) & - & 1200 & 600 \\
$\mathbf{3 3 5}$ & nitrato de amonio & 30 & - & 15 \\
& lombri compost (2) & - & 2000 & 1000 \\
$\mathbf{4 3 2}$ & 18-5-15-6-0,7 & 100 & - & 50 \\
& compost de palma (3) & - & 2500 & 1300 \\
$\mathbf{5 2 3}$ & nitrato de amonio & 45 & - & 23 \\
& lombri compost (2) & - & 3000 & 1500 \\
$\mathbf{6 2 2}$ & 18-5-15-6-0,7 & 110 & - & 55 \\
& lombri compost (2) & - & 3000 & 23 \\
$\mathbf{7 1 9}$ & nitrato de amonio & 45 & - & 1500 \\
\hline
\end{tabular}

dds - días después de la siembra

†(1) compost de la Estación Experimental de Ochomogo - UCR; (2) compost de la Hacienda ganadera Pozo Azul, Río Frío de Sarapiquí, Costa Rica; (3) compost de desecho de palma de aceite.

Cuadro 3. Características de las enmiendas orgánicas que se usaron en la investigación. Estación Experimental Los Diamantes, Limón, Costa Rica. 2003-2005.

\begin{tabular}{|c|c|c|c|c|c|c|c|c|c|c|c|}
\hline \multirow[t]{2}{*}{ Enmienda $\dagger$} & $\mathbf{N}$ & $\mathbf{P}$ & $\mathbf{C a}$ & Mg & $\mathbf{K}$ & $\mathbf{F e}$ & $\mathrm{Cu}$ & $\mathbf{Z n}$ & Mn & humedad & $\begin{array}{c}\$ \mathrm{mgC} . \text { mic. } \\
100 \mathrm{mg}^{-1}\end{array}$ \\
\hline & \multicolumn{5}{|c|}{$\%$} & \multicolumn{4}{|c|}{$\mathrm{mg} / \mathrm{kg}$} & $\%$ & \\
\hline boñiga & 2,40 & 0,49 & 0,63 & 0,28 & 0,34 & 20.200 & 87 & 433 & 623 & 84,2 & - \\
\hline lombri compost (1) & 1,92 & 1,63 & 1,99 & 0,80 & 1,59 & 844 & 69 & 340 & 359 & 63,3 & 4,48 \\
\hline lombri compost (2) & 2,15 & 1,34 & 5,26 & 0,83 & 2,79 & 7.265 & 105 & 382 & 745 & 59,5 & 2,20 \\
\hline lombri compost (2) & 1,33 & 1,07 & 5,15 & 0,79 & 2,24 & 8.397 & 80 & 329 & 800 & 71,8 & 2,20 \\
\hline compost palma (3) & 1,79 & 0,24 & 1,78 & 1,20 & 1,38 & 30.350 & 82 & 91 & 460 & 60,5 & 3,10 \\
\hline
\end{tabular}

† (1) compost de la Estación Experimental de Ochomogo - UCR; (2) compost de Hacienda Pozo Azul, Río Frío; (3) compost de desecho de palma de aceite.

łconcentración de la biomasa microbiana (mg de C microbiano por 100 mg de suelo seco), de acuerdo con la metodología de Vandevivere y Ramírez (1995). 
Cuadro 4. Cantidad de nutrimentos (kg/ha) aplicada por área, de acuerdo con la densidad de población. Estación Experimental Los Diamantes, Limón, Costa Rica. 2003-2005.

\begin{tabular}{|c|c|c|c|c|}
\hline \multirow[b]{2}{*}{ Nutrimentos } & \multirow[b]{2}{*}{ Fertilización } & \multicolumn{3}{|c|}{ Densidad - plantas/ha } \\
\hline & & 3.333 & 5.000 & 6.666 \\
\hline \multirow[t]{3}{*}{$\mathbf{N}$} & química & 127 & 192 & 256 \\
\hline & orgánica & 146 & 221 & 295 \\
\hline & químico-orgánica & 137 & 207 & 276 \\
\hline \multirow{3}{*}{$\mathbf{P} / \mathbf{P}_{2} \mathbf{O}_{5}$} & química & 26 & 39 & 52 \\
\hline & orgánica & 72 & 109 & 145 \\
\hline & químico-orgánica & 49 & 74 & 98 \\
\hline \multirow[t]{3}{*}{$\mathrm{Ca} / \mathrm{CaO}$} & química & 0 & 0 & 0 \\
\hline & orgánica & 290 & 440 & 586 \\
\hline & químico-orgánica & 146 & 221 & 294 \\
\hline \multirow[t]{3}{*}{ Mg/ MgO } & química & 24 & 37 & 49 \\
\hline & orgánica & 62 & 94 & 125 \\
\hline & químico-orgánica & 43 & 66 & 87 \\
\hline \multirow[t]{3}{*}{$\mathrm{K} / \mathrm{K}_{2} \mathrm{O}$} & química & 60 & 91 & 122 \\
\hline & orgánica & 145 & 220 & 293 \\
\hline & químico-orgánica & 103 & 156 & 208 \\
\hline
\end{tabular}

cosechados anteriormente por no haber alcanzado los $10 \mathrm{~cm}$ de diámetro, se realizó a los 20 mds (tercera semana de julio del 2005). Aproximadamente tres meses y medio más tarde (primera semana de noviembre del 2005) se cosecharon todos los rebrotes aptos. En este caso solamente se tomó nota del número de palmitos por planta y por ha.

Los palmitos fueron transportados al laboratorio el mismo día de la cosecha. En la cosecha de febrero, se almacenaron los palmitos en una cámara a $10^{\circ} \mathrm{C}$ y se evaluaron el siguiente día.

Después de remover las vainas externas, se midió el largo, el diámetro y el peso del palmito caulinar y foliar. Cuando el palmito foliar presentaba porciones de diámetros diferentes se midieron el diámetro y el largo de cada porción (Figura 2).

Con estos datos fueron calculados los siguientes parámetros:

a. Diámetro ponderado del palmito foliar (DPPF):

$$
\text { DPPF }=[(\text { DFMGxLPFMG })+(\text { DFMAxLPFMA })] / \text { LPFT; }
$$

Donde:

DFMG = diámetro de la sección más gruesa del palmito foliar;

LPFMG = largo de la sección más gruesa del palmito foliar;

DFMA = diámetro de la sección más angosta del palmito foliar;

LPFMG = largo de la sección más angosta del palmito foliar;

LPFT = largo total del palmito foliar.

b. Relación largo de la sección del palmito foliar con mayor diámetro pelo largo total del palmito foliar (LPFMG/LPFT): se asume que cuanto mayor es el valor de esta relación, mayor es la sección más gruesa y mejor la calidad (presentación) del palmito;

c. Volumen del palmito foliar (VPF): el volumen $\left(\mathrm{cm}^{3}\right)$ fue calculado considerando el palmito como un cilindro y, empleando la fórmula:

$\mathrm{VPF}=\left(\pi \mathrm{x}(\mathrm{DPFP} / 2)^{2} \mathrm{xLPFT}\right)$

d. Densidad del palmito foliar $(\mathrm{dPF})=$ peso del palmito foliar/VPF $\left(\mathrm{g} / \mathrm{cm}^{3}\right)$. 


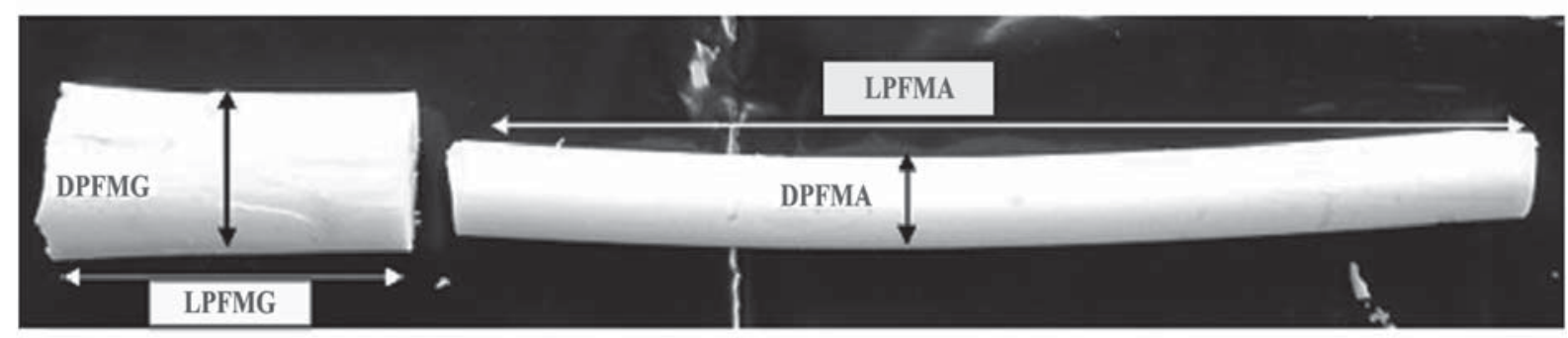

LPFT

Figura 2. Representación esquemática de las medidas hechas al palmito, cuando presentaba secciones con diferente diámetro. Estación Experimental Los Diamantes, Limón, Costa Rica. 2005.

Secciones del palmito foliar: DPFMG = diámetro de la sección más gruesa; DPFMA = diámetro de la sección más angosta; LPFMG = largo de la sección más gruesa; LPFMA = largo de la sección más angosta; LPFT = largo total del palmito.

Los datos experimentales fueron sometidos a un análisis de varianza y a un análisis de correlación, empleando el programa estadístico SAS ${ }^{\circledR}$.

\section{RESULTADOS}

\section{Crecimiento de la planta}

\section{Número de hojas del tallo primario}

En el período de los seis a los 15 meses después de la siembra (mds), el número promedio de hojas varió de $6,7 \pm 1,0$ a $6,2 \pm 0,7$, alcanzando un valor máximo de 7,2 $\pm 0,5$, a los 12 mds; valores inferiores a los observados por Arroyo (2004) (8,7 a 7,3 hojas por planta, en el periodo de los 6 a 12 mds). Por otra parte, Ramos (2002) obtuvo un promedio de 5,8 hojas por planta, a los 15 mds. Esta diferencia se debe principalmente a una mejor fertilización (Bovi et al. 2002; Arroyo 2004); y posiblemente a una condición más seca, en el estudio realizado por Ramos (2002).

El número de hojas por planta no respondió a las prácticas culturales utilizadas, excepto cuando se evaluó esta variable a los 6 mds. Asimismo, se observó una interacción densidad $\mathrm{x}$ arreglo de siembra $(\mathrm{P}=0,0001)$ solamente a los $6 \mathrm{mds}$, lo que pone en evidencia que al poseer las palmas una arquitectura estrictamente simétrica, no son capaces de compensar la competencia intraespecífica (entre plantas de la misma especie), mediante la reducción en el número de ejes o tallos. Por lo tanto, su tasa de producción de hojas cambia muy poco en un amplio rango de densidades de población. El número de hojas por planta se manutuvo relativamente constante hasta los $12 \mathrm{mds}$, para luego disminuir a los 15 mds (Cuadro 5).

\section{Altura del tallo primario}

La altura promedio del tallo primario aumentó de $19,3 \pm 4,0$ a $142,2 \pm 20,6 \mathrm{~cm}$, entre los 6 y 15 mds. Este crecimiento en altura es menor que la descrita por Arroyo (2004), quien obtuvo, con este mismo cultivar y en la misma Estación Experimental, un valor promedio de 152,3 cm de altura, a los 12 mds. Esta diferencia se debe, posiblemente a mayor cantidad de fertilizantes utilizada. En nuestro experimento no hubo respuesta de la altura del tallo, en la primera cosecha, a los tratamientos experimentales evaluados (Cuadro 5).

\section{Diámetro basal $(D B)$ del tallo primario}

El diámetro basal (DB) promedio del tallo primario

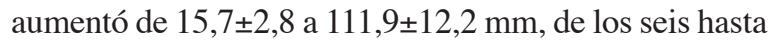
los 15 mds, en concordancia con otras observaciones 


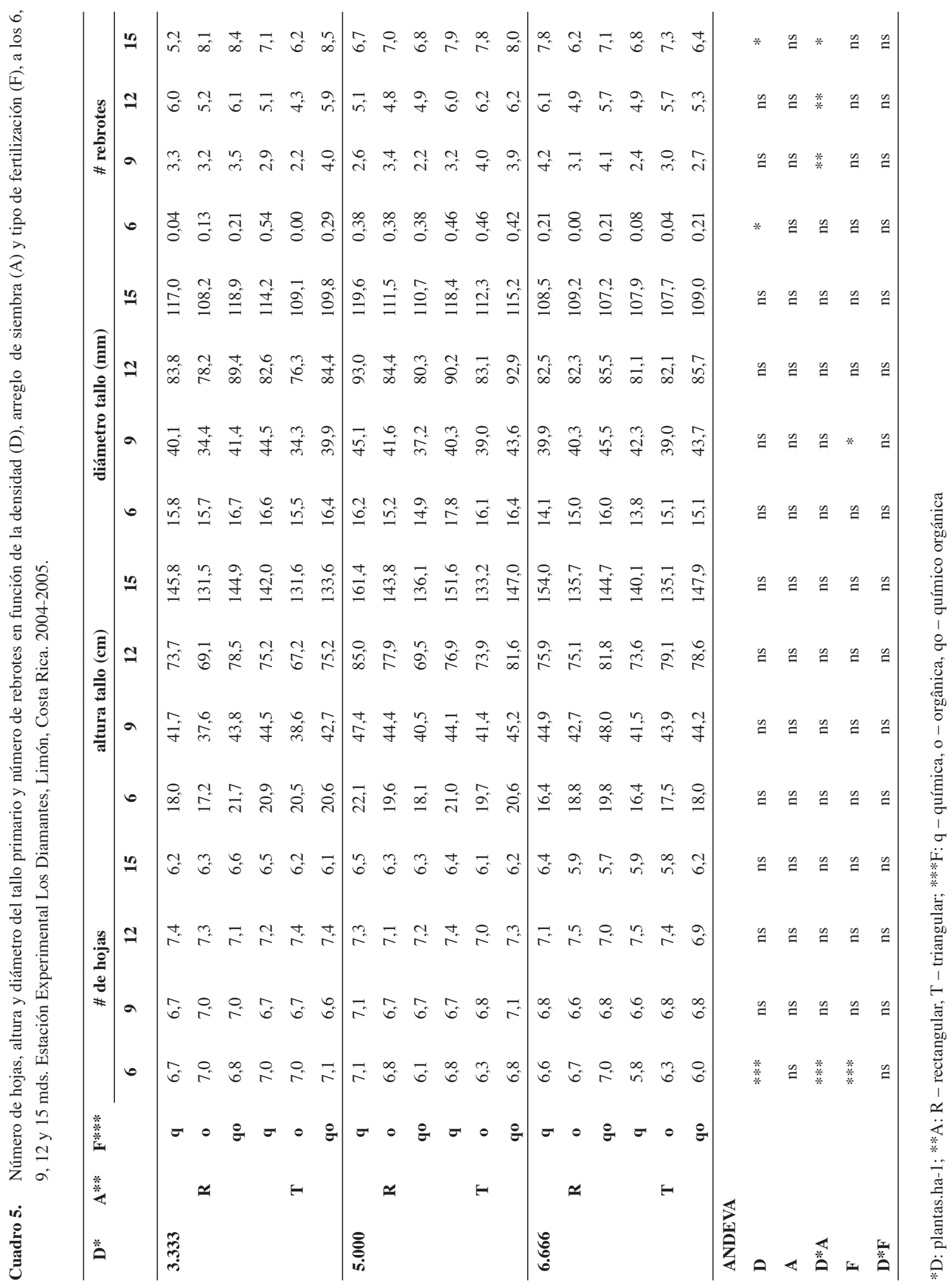


(Arroyo 2004) realizadas en la misma finca experimental. Se observó un pequeño efecto del tipo de fertilización en el DB, a los 9 mds ( $\mathrm{P}=0$,0304) (Cuadro 5).

Número de rebrotes por cepa y porcentaje deplantas con rebrotes

El número de rebrotes es una característica fundamental, que indica la capacidad de regeneración de la plantación, y generalmente es muy afectado por la densidad de siembra, como respuesta a la disponibilidad de luz. El número promedio de rebrotes por cepa incrementó de $0,3 \pm 0,4$ a 7,3 $\pm 1,3$ en el período de los seis hasta los 15 mds. Las parcelas con una densidad de siembra de 3.333 plantas/ha mostraron un incremento en el número de rebrotes a los seis y a los $15 \mathrm{mds}(\mathrm{P}$ $=0,032$ y 0,0374, respectivamente) (Cuadro 5).

Bogantes (2003) observó un promedio de $1,95 \pm 0,51$ y $1,0 \pm 0,4$ rebrotes por planta, a los $9 \mathrm{mds}$, en plantaciones con densidades de 6.666 y 5.000 plantas/ha, respectivamente. Valores inferiores a los que se obtuvieron en este trabajo. Sin embargo, Arroyo (2004) obtuvo un promedio de 7,4 rebrotes por planta, a los 12 mds para el mismo cultivar Diamantes 10 . Aunque ese autor utilizó una densidad de plantación de 10.000 plantas/ha, el recuento de rebrotes fue hecho en plantas con raíz desnuda (que permite identificar rebrotes incipientes) y se aplicó mayor cantidad de fertilizantes químicos. Ramos (2002) también observó un promedio de rebrotes ligeramente superior a los observados en nuestra investigación $(7,7$ rebrotes por planta, a los $15 \mathrm{mds}$ ), con una densidad de siembra de 5.000 plantas/ha.

El tipo de fertilización no influyó en el número de rebrotes por cepa (Cuadro 5). Sin embargo, Vega et al. (2004), empleando dosis crecientes de biosólido (equivalentes a 0, 100, 200 y $400 \mathrm{~kg} /$ ha de N), observaron un mayor número de rebrotes en las plantas que recibieron mayor cantidad de nitrógeno.

El porcentaje promedio de plantas con rebrotes, a los 6 mds, fue de $13,8 \%$. No obstante, a los 9 mds, un $86,3 \%$ de las plantas útiles ya presentaban rebrotes y, a partir de los 12 mds, casi el $100 \%$ de las plantas pre- sentaban rebrotes. Resultados similares fueron publicados por Arroyo (2004). La densidad, disposición de siembra y el tipo de fertilización no influyeron sobre esta variable, en ninguno de los períodos evaluados.

Evaluaciones fenológicas de los rebrotes después de la cosecha

Aproximadamente tres meses después de la cosecha del tallo primario (20 mds), el número promedio de rebrotes pequeños $(\mathrm{RBP})$, con un diámetro inferior a $3 \mathrm{~cm}$, fue de 4,1 por cepa; mientras que, el número promedio de rebrotes grandes (RBG), con un diámetro mayor a $3 \mathrm{~cm}$, fue de 2,8 por cepa. Sin embargo, en la segunda evaluación (23 mds), el número promedio de rebrotes pequeños y grandes fue el inverso de lo observado tres meses antes, esto es, 2,5 y 4,4 (Cuadro 6).

La fertilización no influyó en el número de rebrotes pequeños pero si en el número de rebrotes grandes, a los $20 \mathrm{mds}(\mathrm{P}=0,0103)$ y $23 \mathrm{mds}(\mathrm{P}=0,0002)$. Las plantas fertilizadas con abono químico y químico-orgánico presentaron un mayor número de rebrotes grandes que aquellas que recibieron solamente fertilizante orgánico (Figura 3). Las cepas de las parcelas con menor

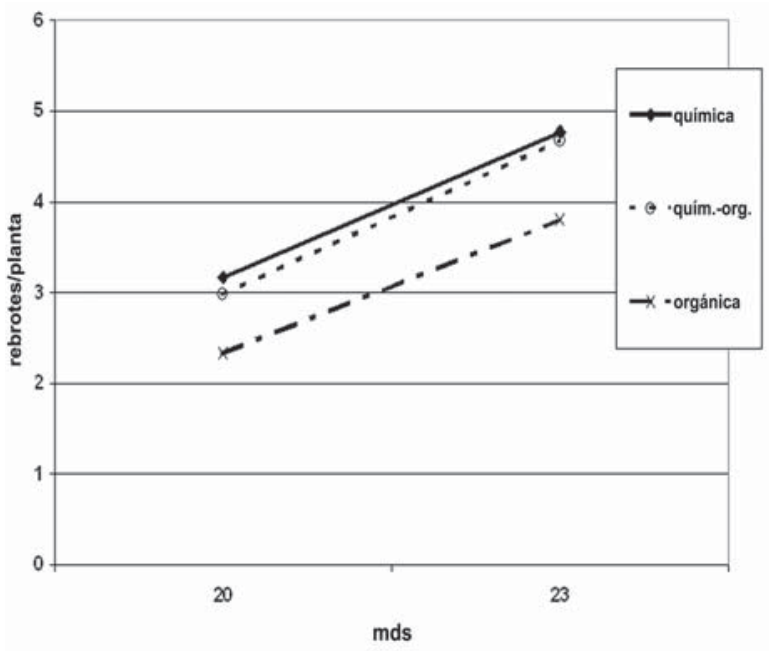

Figura 3. Número de rebrotes grandes (diámetro $>3 \mathrm{~cm}$ ) por planta en función del tipo de fertilización, a los 20 y 23 mds. Estación Experimental Los Diamantes, Limón, Costa Rica. 2005. 
Cuadro 6. Número de rebrotes pequeños (diámetro $<3 \mathrm{~cm}$ ) y grandes (diámetro $>3 \mathrm{~cm}$ ), y de hojas; altura y diámetro de los rebrotes grandes, en función de la densidad, arreglo de siembra y tipo de fertilización, a los 20 y 23 mds. Estación Experimental Los Diamantes, Limón, Costa Rica. 2004-2005.

\begin{tabular}{|c|c|c|c|c|c|c|c|c|c|c|c|c|}
\hline \multirow[t]{2}{*}{ D* } & \multirow[t]{2}{*}{$\mathbf{A}^{* *}$} & \multirow[t]{2}{*}{$\mathbf{F}^{* * *}$} & \multicolumn{2}{|c|}{ \#RBP } & \multicolumn{2}{|c|}{ \#RBG } & \multicolumn{2}{|c|}{ \# hojas } & \multicolumn{2}{|c|}{ Altura (cm) } & \multicolumn{2}{|c|}{$\begin{array}{c}\text { diámetro } \\
(\mathbf{m m})\end{array}$} \\
\hline & & & 20 & 23 & 20 & 23 & 20 & 23 & 20 & 23 & 20 & 23 \\
\hline \multirow[t]{6}{*}{3.333} & & $\mathbf{q}$ & 3,6 & 3,0 & 4,0 & 5,4 & 4,8 & 5,1 & 74,5 & 141,8 & 48,3 & 87,8 \\
\hline & $\mathbf{R}$ & $\mathbf{o}$ & 3,5 & 2,3 & 3,8 & 5,5 & 4,3 & 5,0 & 66,4 & 125,0 & 48,8 & 80,7 \\
\hline & & qo & 3,6 & 1,7 & 3,8 & 4,9 & 4,6 & 4,8 & 79,2 & 134,7 & 55,4 & 82,5 \\
\hline & $\mathbf{T}$ & $\mathbf{q}$ & 4,6 & 2,7 & 2,6 & 5,1 & 5,0 & 5,0 & 71,9 & 137,6 & 51,7 & 81,1 \\
\hline & $\mathbf{T}$ & o & 4,5 & 2,6 & 1,5 & 2,9 & 4,7 & 5,1 & 64,3 & 103,6 & 45,4 & 74,0 \\
\hline & & qo & 4,9 & 2,3 & 2,7 & 5,7 & 4,6 & 4,7 & 65,7 & 123,9 & 47,1 & 76,5 \\
\hline \multirow[t]{6}{*}{5.000} & & $\mathbf{q}$ & 3,0 & 1,8 & 2,8 & 4,5 & 4,5 & 5,0 & 70,8 & 141,7 & 49,0 & 84,3 \\
\hline & $\mathbf{R}$ & $\mathbf{o}$ & 3,9 & 1,9 & 2,7 & 4,2 & 4,3 & 4,5 & 64,8 & 120,6 & 43,5 & 74,9 \\
\hline & & qo & 4,7 & 3,1 & 2,5 & 3,4 & 5,1 & 4,5 & 81,8 & 106,9 & 53,8 & 64,9 \\
\hline & $\mathbf{T}$ & $\mathbf{q}$ & 3,6 & 2,8 & 4,4 & 4,9 & 4,6 & 4,7 & 81,8 & 131,4 & 53,3 & 83,0 \\
\hline & $\mathbf{T}$ & $\mathbf{o}$ & 4,1 & 3,0 & 2,2 & 3,5 & 4,5 & 5,5 & 64,6 & 125,6 & 48,2 & 81,9 \\
\hline & & qo & 4,3 & 2,7 & 3,2 & 4,9 & 4,3 & 4,2 & 72,4 & 125,3 & 48,4 & 75,0 \\
\hline \multirow[t]{6}{*}{6.666} & & $\mathbf{q}$ & 4,3 & 2,8 & 2,8 & 4,7 & 4,5 & 4,9 & 80,2 & 151,9 & 55,7 & 85,7 \\
\hline & $\mathbf{R}$ & o & 3,0 & 2,5 & 2,0 & 2,7 & 4,7 & 4,8 & 64,1 & 122,4 & 44,3 & 81,6 \\
\hline & & qo & 4,5 & 2,7 & 2,5 & 4,3 & 4,4 & 4,7 & 63,5 & 116,9 & 43,2 & 75,6 \\
\hline & $\mathbf{T}$ & $\mathbf{q}$ & 5,0 & 3,4 & 2,5 & 4,0 & 4,8 & 5,1 & 70,3 & 140,0 & 47,1 & 79,4 \\
\hline & $\mathbf{T}$ & o & 4,5 & 2,3 & 1,8 & 4,0 & 4,6 & 4,6 & 61,3 & 125,4 & 43,2 & 72,5 \\
\hline & & qo & 4,3 & 2,0 & 2,5 & 4,7 & 4,5 & 4,6 & 69,1 & 133,4 & 43,7 & 79,2 \\
\hline D & & & $\mathrm{ns}$ & $\mathrm{ns}$ & $\mathrm{ns}$ & $*$ & $\mathrm{~ns}$ & $\mathrm{~ns}$ & $\mathrm{~ns}$ & $\mathrm{~ns}$ & ns & $\mathrm{ns}$ \\
\hline A & & & $\mathrm{ns}$ & ns & $\mathrm{ns}$ & $\mathrm{ns}$ & $\mathrm{ns}$ & $\mathrm{ns}$ & $\mathrm{ns}$ & ns & ns & $\mathrm{ns}$ \\
\hline $\mathbf{D}^{*} \mathbf{A}$ & & & ns & ns & $\mathrm{ns}$ & $\mathrm{ns}$ & ns & ns & ns & $\mathrm{ns}$ & ns & ns \\
\hline $\mathbf{F}$ & & & ns & ns & $* *$ & $* * *$ & $\mathrm{~ns}$ & $*$ & $* *$ & $* * *$ & ns & $*$ \\
\hline $\mathbf{D} * \mathbf{F}$ & & & $\mathrm{ns}$ & $\mathrm{ns}$ & $\mathrm{ns}$ & ns & ns & ns & ns & $\mathrm{ns}$ & ns & ns \\
\hline
\end{tabular}

*D = densidad (plantas/ha); **A = arreglo: $\mathrm{R}$ - rectangular; $\mathrm{T}-$ triangular (pata de gallo); ***F = fertilización: $\mathrm{q}-$ química, o - orgánica, qo - químico-orgánica; $\mathrm{RBP}=$ rebrotes pequeños (diámetro $<3 \mathrm{~cm}$.), $\mathrm{RBG}=$ rebrotes más grandes (diámetro $>3 \mathrm{~cm}$.)

$\mathrm{ns}=(\mathrm{P}>0,05), *=(\mathrm{P}<0,05), * *(\mathrm{P}<0,01), * * *=(\mathrm{P}<0,001)$

densidad de población también presentaron mayor número de rebrotes grandes $(\mathrm{P} \leq 0,0154)$, a los 23 mds.

Los rebrotes de las cepas con fertilización químico-orgánica presentaron menor número de hojas $(4,6 \pm 0,6)$, en comparación con aquellas con fertilización química $(5,0 \pm 0,5)$ y orgánica $(4,9 \pm 0,7)(\mathrm{P}=$ $0,0453)$.

La altura promedio de los rebrotes grandes (ARBG) fue de 70,4 y 128,2 cm, a los 20 y 23 mds. El tipo de fertilización mostró su efecto en esta característica en los dos períodos de evaluación (20 mds: $\mathrm{P}=$ 0,0096; y 23 mds: $\mathrm{P}=0,0007)$. En la primera evaluación, los rebrotes de las cepas fertilizadas con abono químico y quimico-orgánico presentaron una mayor ARBG; mientras que, a los 23 mds, solamente los rebrotes de las cepas fertilizadas con abono químico presentaron mayor altura del tallo (Figura 4).

El diámetro promedio de los rebrotes grandes (DRG) fue de 48,3 y 79,1 mm, a los 20 y 23 mds, 


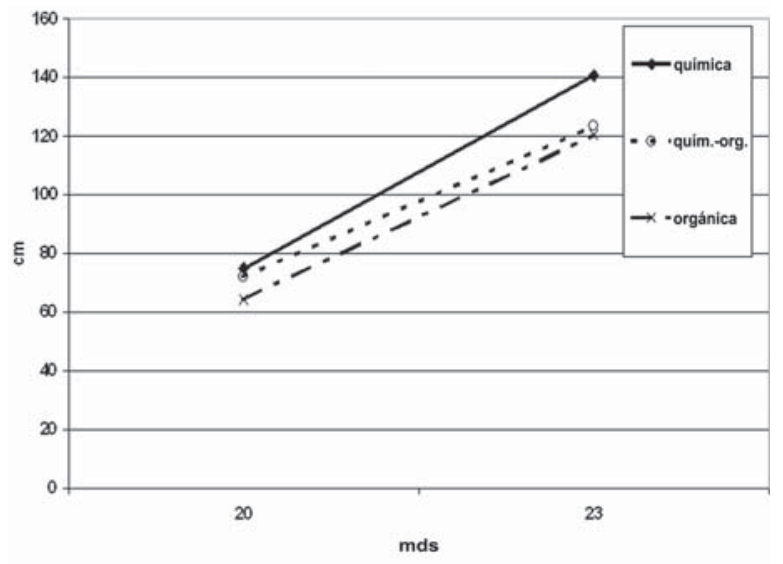

Figura 4. Altura de los rebrotes grandes (diámetro $>3 \mathrm{~cm}$ ) en función del tipo de fertilización, a los 20 y 23 mds. Estación Experimental Los Diamantes, Limón, Costa Rica. 2005.

respectivamente. El efecto del tipo de fertilización también se observó en el DRG (P=0,0258), en la segunda evaluación. Las cepas fertilizadas con abono químico presentaron rebrotes con mayor diámetro promedio $(84,2 \pm 8,8 \mathrm{~mm})$, comparado a los rebrotes de plantas fertilizadas con enmienda orgánica $(77,6 \pm 14,5 \mathrm{~mm})$ y abono químico-orgánico $(75,6 \pm 14,3 \mathrm{~mm})$.

\section{Cosecha del palmito}

El porcentaje promedio de tallos primarios cosechados a los 16,5 mds (febrero del 2005) fue de 78,5\%. En julio del mismo año (21,6 mds) se cosechó el palmito del tallo primario de las cepas con crecimiento retrasado. Esto fue equivalente al 6,5\% del total de las plantas útiles. La pérdida debida a la mortalidad de plantas atribuible al ataque de picudo (Metamasius hemipterus), de Erwinia carotovora u otras causas desconocidas, fue de un $15 \%$ del total de plantas útiles.

A los 23 mds, un promedio de $37 \%$ de las cepas presentaban rebrotes aptos para ser cosechados. La mayor proporción de rebrotes cosechables se presentó en las parcelas con fertilización química $(\mathrm{P}=0,0422)$. Sin embargo, se observó una excepción en función del efecto de la interacción entre la fertilización y la densidad de población $(\mathrm{P}<0,05)$ (Figura 5). En las parcelas con fertilización química el porcentaje de plantas cosechadas decreció con el incremento en la densidad; mientras que

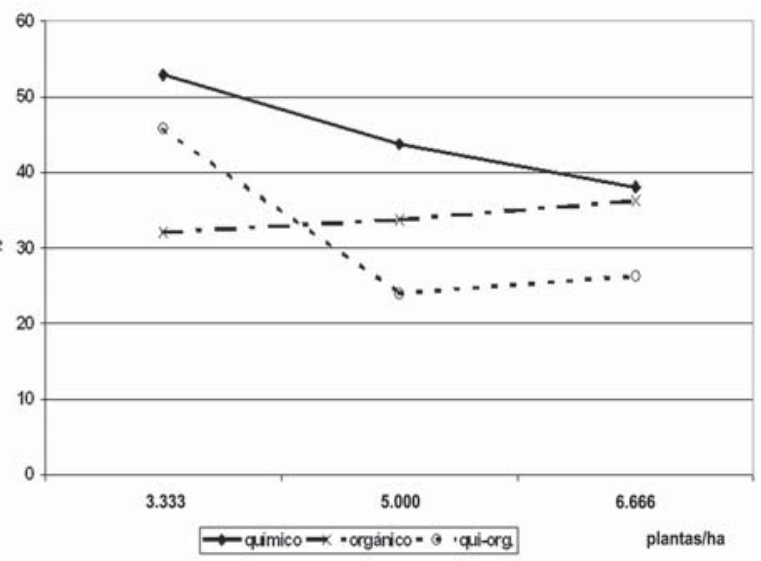

Figura 5. Porcentaje de cepas con rebrotes aptos para el corte a los 23 mds, en función del tipo de fertilización y la densidad de población. Estación Experimental Los Diamantes, Limón, Costa Rica. 2005.

en las parcelas con fertilización orgánica se observó lo inverso. En la densidad más alta el porcentaje de plantas cosechadas fue similar en las parcelas con fertilización química y orgánica; en tanto que en las parcelas con fertilización químico-orgánica, el porcentaje disminuyó con el aumento de densidad, de aquella de 3.333 plantas/ha hacia las densidades de 5.000 y 6.666 plantas/ha que mostraron similar porcentaje.

La densidad, la disposición de siembra y el tipo de fertilización no influyeron en el número de rebrotes cosechados por cepa y por área, a los 25 mds (Cuadro 7). Sin embargo, se observó una tendencia (aunque no apoyada estadísticamente) a la disminución en el número de rebrotes cosechados por cepa con el incremento en la densidad de la plantación. Una tendencia inversa fue observada en relación con el número de palmitos cortados por hectárea, ya que el número de palmitos cosechados se incrementó con el aumento en la densidad de siembra. De manera similar, Bogantes (2003) observó una disminución en el número de palmitos por cepa, con una tendencia curvilínea, y un aumento en el número de palmitos por área, con el incremento en la densidad de siembra de 5.000 a 20.000 plantas/ha.

\section{Palmito caulinar}

No se observó respuesta alguna en las características del palmito caulinar a las prácticas culturales 
Cuadro 7. Número de palmitos cosechados por cepa y por hectárea, en función de la densidad, arreglo de siembra y tipo de fertilización, a los 25 mds. Estación Experimental Los Diamantes, Limón, Costa Rica. 2004-2005.

\begin{tabular}{|c|c|c|c|c|}
\hline $\begin{array}{c}\text { densidad } \\
\text { plantas/ } \\
\text { ha }\end{array}$ & arreglo & $\begin{array}{l}\text { fertiliza- } \\
\text { ción }\end{array}$ & $\begin{array}{l}\text { palmi- } \\
\text { tos/cepa }\end{array}$ & $\begin{array}{l}\text { palmi- } \\
\text { tos/ha }\end{array}$ \\
\hline \multirow{6}{*}{3.333} & \multirow{3}{*}{ rectangular } & química & 1,5 & 5.006 \\
\hline & & orgánica & 1,2 & 3.859 \\
\hline & & $\begin{array}{l}\text { químico-or- } \\
\text { gánica }\end{array}$ & 1,2 & 4.125 \\
\hline & \multirow{3}{*}{ triangular } & química & 1,4 & 4.505 \\
\hline & & orgánica & 1,3 & 4.464 \\
\hline & & $\begin{array}{l}\text { químico-or- } \\
\text { gánica }\end{array}$ & 1,8 & 5.831 \\
\hline \multirow{6}{*}{5.000} & \multirow{3}{*}{ rectangular } & química & 1,3 & 6.677 \\
\hline & & orgánica & 1,3 & 6.260 \\
\hline & & $\begin{array}{l}\text { químico-or- } \\
\text { gánica }\end{array}$ & 1,1 & 5.638 \\
\hline & \multirow{3}{*}{ triangular } & química & 1,1 & 5.626 \\
\hline & & orgánica & 1,3 & 6.596 \\
\hline & & $\begin{array}{l}\text { químico-or- } \\
\text { gánica }\end{array}$ & 1,2 & 6.162 \\
\hline \multirow{6}{*}{6.666} & \multirow{3}{*}{ rectangular } & química & 0,9 & 5.992 \\
\hline & & orgánica & 0,8 & 5.369 \\
\hline & & $\begin{array}{l}\text { químico-or- } \\
\text { gánica }\end{array}$ & 1,0 & 6.458 \\
\hline & \multirow{3}{*}{ triangular } & química & 1,2 & 7.676 \\
\hline & & orgánica & 0,8 & 5.430 \\
\hline & & $\begin{array}{l}\text { químico-or- } \\
\text { gánica }\end{array}$ & 1,0 & 6.511 \\
\hline \multicolumn{5}{|l|}{ ANDEVA } \\
\hline D & & & ns & ns \\
\hline $\mathbf{A}$ & & & ns & ns \\
\hline $\mathbf{D}^{*} \mathbf{A}$ & & & ns & ns \\
\hline $\mathbf{F}$ & & & ns & ns \\
\hline $\mathbf{D}^{* \mathbf{F}}$ & & & ns & ns \\
\hline
\end{tabular}

$\mathrm{ns}=(\mathrm{P}>0,05)$.

evaluadas, a los 16 mds. Lo que si se observó fue que el largo $(9,7 \pm 2,4 \mathrm{~cm})$ y el peso $(212,3 \pm 79,4 \mathrm{~g})$ promedio del palmito caulinar cortado en julio (25 mds) fue inferior al de la primera cosecha, ya que era un material oriundo de plantas más viejas y, por lo tanto, más lignificadas, además de ser cortado en condiciones de menor humedad. El diámetro promedio $(54,4 \pm 5,8$ $\mathrm{mm}$ ) del segundo corte si fue similar al de la primera cosecha. El rendimiento promedio de palmito caulinar fue de $1.247 \mathrm{~kg} / \mathrm{ha}$ y se incrementó con el aumento en la densidad de siembra $(\mathrm{P}=0,0001)$ (Cuadro 8).

\section{Palmito foliar}

Un aspecto de primordial relevancia en la comercialización del palmito foliar al natural es su diámetro y su longitud. Un palmito más grueso tiene mejor presentación y mejor aceptación.

El diámetro promedio del palmito foliar más grueso (DPFMG), de la primera cosecha (16 mds), fue de 32,8 $\pm 3,9 \mathrm{~mm}$; y el menor diámetro se obtuvo en la mayor densidad de siembra (Cuadro 9). El palmito cosechado en julio (a los 20 mds) presentó un DPFMG

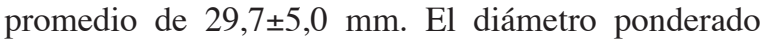
promedio del palmito foliar (DPFP) fue de 28,0 $\pm 2,4$ $\mathrm{mm}$, y los tratamientos experimentales no influyeron en esta característica. El palmito cosechado en julio presentó un DPFP promedio de 27,6 $\pm 3,1 \mathrm{~mm}$, similar al observado anteriormente. Investigaciones recientes (Yuyama 2005), han mostrado valores mayores en el diámetro promedio del palmito proveniente de cepas sembradas a menor densidad (3.333 plantas/ha), comparado con el palmito producido en cepas con densidades de 5.000 y 10.000 plantas/ha.

El largo promedio del palmito foliar más grueso (LPFMG) a los 16 mds fue de 27,4 7,1 cm, mientras que el palmito cosechado de los tallos primarios no cosechados anteriormente, presentó un LPFMG promedio de $35,2 \pm 11,3 \mathrm{~cm}$ a los $20 \mathrm{mds}$. El largo total promedio del palmito foliar (LPFT) fue similar en ambas cosechas $(42,9 \pm 3,4 \mathrm{~cm}$ y $43,7 \pm 9,0 \mathrm{~cm}$, en la primera y segunda cosecha, respectivamente). De nuevo, la densidad, el arreglo de siembra y el tipo de fertilización no influyeron en estas variables (Cuadro 9). La relación (LPFMG/LPFT) entre el largo de la sección del palmito foliar de mayor diámetro (LFM) y el largo total promedio del palmito foliar (LPFT) presentó un promedio de $0,6 \pm 0,2$. Tampoco los tratamientos influyeron sobre esta característica.

El peso promedio del palmito foliar (PPF) fue de $275,4 \pm 56,3$ g y se incrementó de la menor densidad de 
Cuadro 8. Diámetro (DPC), largo (LPC), peso (PPC) y rendimiento (RPC) del palmito caulinar, en función de la densidad y el arreglo espacial y el tipo de fertilización a los 16 mds. Estación Experimental Los Diamantes, Limón, Costa Rica. 2004-2005.

\begin{tabular}{|c|c|c|c|c|c|c|}
\hline $\begin{array}{l}\text { Densidad } \\
\text { plantas/ha }\end{array}$ & Arreglo & Fertilización & $\begin{array}{l}\text { DPC } \\
(\mathrm{mm})\end{array}$ & $\begin{array}{l}\text { LPC } \\
\text { (cm) }\end{array}$ & PPC (g) & $\begin{array}{c}\text { RPC } \\
\text { (kg/ha) }\end{array}$ \\
\hline \multirow{6}{*}{3.333} & rectangular & química & 55,4 & 13,9 & 323,0 & 954,0 \\
\hline & & orgánica & 64,0 & 13,6 & 326,1 & 829,5 \\
\hline & & químico-org. & 55,2 & 13,7 & 345,9 & 1076,6 \\
\hline & triangular & química & 54,2 & 12,5 & 319,4 & 754,9 \\
\hline & & orgánica & 58,5 & 14,7 & 390,4 & 927,2 \\
\hline & & químico-org. & 54,8 & 14,6 & 345,8 & 734,9 \\
\hline \multirow{6}{*}{5.000} & rectangular & química & 56,4 & 16,2 & 423,6 & 1888,9 \\
\hline & & orgánica & 56,6 & 15,2 & 378,4 & 1766,0 \\
\hline & & químico-org. & 55,0 & 13,5 & 327,5 & 1387,1 \\
\hline & triangular & química & 56,6 & 14,9 & 393,7 & 1681,7 \\
\hline & & orgánica & 53,6 & 13,4 & 316,1 & 1313,9 \\
\hline & & químico-org. & 53,9 & 13,9 & 316,2 & 1278,9 \\
\hline \multirow{6}{*}{6.666} & rectangular & química & 54,6 & 16,6 & 386,6 & 1875,0 \\
\hline & & orgánica & 53,9 & 13,2 & 297,6 & 1279,2 \\
\hline & & químico-org. & 53,9 & 14,9 & 342,8 & 2079,9 \\
\hline & triangular & química & 54,9 & 14,0 & 315,1 & 1661,4 \\
\hline & & orgánica & 54,7 & 14,0 & 322,6 & 1436,5 \\
\hline & & químico-org. & 55,0 & 14,8 & 346,1 & 1863,6 \\
\hline ANDEVA & & & ns & ns & ns & $* *$ \\
\hline D & & & ns & ns & ns & ns \\
\hline $\mathbf{A}$ & & & ns & ns & ns & ns \\
\hline $\mathbf{D} * \mathbf{A}$ & & & ns & ns & ns & ns \\
\hline $\mathbf{F}$ & & & ns & ns & ns & ns \\
\hline $\mathbf{D} * \mathbf{F}$ & & & & & & \\
\hline
\end{tabular}

$\mathrm{ns}=(\mathrm{P} \geq 0,05), *=(\mathrm{P} \leq 0,05), * *(\mathrm{P} \leq 0,01)$.

siembra a la densidad intermedia, pero disminuyó con la densidad más alta (Cuadro 9). El palmito cosechado en julio presentó un PPF promedio de 263,9 $\pm 68,8 \mathrm{~cm}$. Yuyama (2005) observó un mayor peso promedio del palmito proveniente de cepas sembradas a una densidad de 3.333 plantas/ha, comparado con el palmito producido por cepas a mayor densidad (5.000 y 10.000 plantas/ha). Sin embargo, Clement et al. (1996) no obtuvieron diferencias en el peso del palmito con las mismas densidades evaluadas en el presente trabajo (3.333, 5.000 y 6.666 plantas/ha). El rendimiento promedio de palmito foliar fue de $986,3 \mathrm{~kg} / \mathrm{ha}$ y fue creciente con el aumento en la densidad de siembra $(\mathrm{P}=0,0001)$.

Correlación entre los parámetros fenológicos y las características de producción del palmito

El diámetro del palmito caulinar (DPC) se correlacionó positivamente con el número de hojas $(\mathrm{NH})$, la altura (AT) y el diámetro (DB) del tallo primario; el NH fue el mejor estimador de esta importante variable. El largo del palmito caulinar (LPC) se correlacionó 
Cuadro 9. Diámetro del palmito foliar mas grueso (DPFMG), diámetro del palmito foliar ponderado (DPFP), largo del palmito foliar mas grueso (LPFMG) y largo total del palmito foliar (LPFT), relación LPFMG/LPFT (LPFM/T), volumen (VPF), peso (PPF) del palmito foliar, y rendimiento del palmito foliar (RPF) en función de la densidad, el arreglo de siembra y el tipo de fertilización. Estación Experimental Los Diamantes, Limón, Costa Rica. 2004-2005.

\begin{tabular}{|c|c|c|c|c|c|c|c|c|c|c|}
\hline \multirow[t]{2}{*}{ D } & \multirow[t]{2}{*}{$\mathbf{A}$} & \multirow[t]{2}{*}{$\mathbf{F}$} & DPFMG & DPFP & LPFMG & LPFT & \multirow[t]{2}{*}{ LPFM/T } & \multirow{2}{*}{$\begin{array}{l}\text { VPF } \\
\mathbf{c m}^{3}\end{array}$} & \multirow{2}{*}{$\begin{array}{c}\text { PPF } \\
(\mathrm{g})\end{array}$} & \multirow{2}{*}{$\begin{array}{c}\text { RPF } \\
\text { (kg/ha) }\end{array}$} \\
\hline & & & \multicolumn{2}{|c|}{$\mathbf{m m}$} & \multicolumn{2}{|c|}{$\mathbf{c m}$} & & & & \\
\hline \multirow{6}{*}{3.333} & \multirow{4}{*}{$\mathbf{R}$} & $\mathbf{q}$ & 31,9 & 27,8 & 30,5 & 42,9 & 0,7 & 243,5 & 277,2 & 812,7 \\
\hline & & $\mathbf{o}$ & 32,9 & 27,5 & 26,3 & 44,5 & 0,6 & 202,6 & 283,4 & 739,5 \\
\hline & & qo & 34,8 & 28,6 & 25,6 & 44,2 & 0,6 & 233,5 & 322,0 & 971,9 \\
\hline & & $\mathbf{q}$ & 33,3 & 28,4 & 24,1 & 40,4 & 0,6 & 212,6 & 265,7 & 619,8 \\
\hline & \multirow[t]{3}{*}{$\mathbf{T}$} & $\mathbf{o}$ & 34,4 & 28,8 & 24,5 & 42,9 & 0,6 & 221,9 & 291,4 & 636,2 \\
\hline & & qo & 31,9 & 27,2 & 27,6 & 42,3 & 0,7 & 204,4 & 268,2 & 606,1 \\
\hline \multirow{6}{*}{5.000} & & $\mathbf{q}$ & 33,2 & 29,1 & 30,0 & 43,2 & 0,6 & 254,5 & 293,7 & $1.292,1$ \\
\hline & \multirow[t]{3}{*}{$\mathbf{R}$} & $\mathbf{o}$ & 32,2 & 26,5 & 24,0 & 40,5 & 0,6 & 196,6 & 245,0 & $1.124,9$ \\
\hline & & qo & 35,6 & 30,0 & 27,9 & 46,2 & 0,6 & 254,3 & 336,6 & $1.349,5$ \\
\hline & & $\mathbf{q}$ & 35,0 & 29,5 & 26,3 & 44,1 & 0,6 & 256,3 & 344,6 & $1.356,8$ \\
\hline & \multirow[t]{3}{*}{$\mathbf{T}$} & $\mathbf{o}$ & 33,9 & 28,6 & 27,2 & 44,3 & 0,6 & 244,1 & 298,0 & $1.206,1$ \\
\hline & & qo & 33,0 & 28,1 & 28,5 & 44,7 & 0,6 & 241,0 & 290,0 & $1.169,4$ \\
\hline \multirow{6}{*}{6.666} & & $\mathbf{q}$ & 32,1 & 28,1 & 29,7 & 43,3 & 0,7 & 239,3 & 275,4 & $1.430,0$ \\
\hline & \multirow[t]{3}{*}{$\mathbf{R}$} & o & 30,6 & 26,3 & 29,1 & 43,1 & 0,7 & 208,1 & 254,3 & $1.103,6$ \\
\hline & & qo & 30,3 & 26,8 & 31,3 & 42,4 & 0,7 & 214,5 & 252,8 & $1.471,0$ \\
\hline & & $\mathbf{q}$ & 31,3 & 27,1 & 28,0 & 40,9 & 0,7 & 212,3 & 256,5 & $1.334,0$ \\
\hline & \multirow[t]{2}{*}{$\mathbf{T}$} & o & 30,6 & 26,8 & 26,2 & 40,3 & 0,7 & 186,3 & 234,5 & $1.040,9$ \\
\hline & & qo & 33,7 & 28,7 & 26,5 & 41,3 & 0,6 & 227,2 & 274,2 & $1.441,0$ \\
\hline \multicolumn{11}{|c|}{ ANDEVA } \\
\hline \multicolumn{3}{|c|}{ D } & $* *$ & ns & ns & ns & ns & ns & $*$ & $* * *$ \\
\hline \multicolumn{3}{|l|}{ A } & ns & ns & ns & ns & ns & ns & ns & ns \\
\hline \multicolumn{3}{|l|}{$\mathbf{D} * \mathbf{A}$} & ns & ns & ns & ns & ns & ns & ns & ns \\
\hline \multicolumn{3}{|l|}{$\mathbf{F}$} & ns & ns & $\mathrm{ns}$ & ns & ns & $\mathrm{ns}$ & ns & ns \\
\hline \multicolumn{3}{|l|}{$\mathbf{D} * \mathbf{F}$} & ns & ns & ns & ns & ns & ns & $\mathrm{ns}$ & ns \\
\hline
\end{tabular}

$\mathrm{D}=$ densidad (plantas.ha $\left.{ }^{-1}\right) ; \mathrm{A}=$ arreglo: $\mathrm{R}$ - rectangular; $\mathrm{T}$ - triangular; $\mathrm{F}=$ fertilización: $\mathrm{q}$ - química, o - orgánica, qo - químico-orgánica;

$\mathrm{ns}=(\mathrm{P}>0,05), *=(\mathrm{P}<0,05), * *(\mathrm{P}<0,01), * * *=(\mathrm{P}<0,001)$

con el NH y la AT, siendo la altura del tallo primario su mejor estimador. El NH y la AT, además del DB, también se correlacionaron con el peso del palmito caulinar (PPC), aunque las dos primeras variables estimaron mejor el PPC.
Todos los parámetros fenológicos se correlacionaron positivamente con el diámetro del palmito foliar (DPF), con el largo del palmito foliar (LPF) y con el peso del palmito foliar (PPF) (Cuadro 10). Esta observación sirve de aval al uso de estas variables fenológicas 
Cuadro 10. Matriz de correlación entre parámetros fenológicos (diámetro del tallo, DB; número de hojas, $\mathrm{NH}$; altura de la planta, AP), y características del rendimiento del palmito caulinar y foliar. Estación Experimental Los Diamantes, Limón, Costa Rica. 2004-2005.

\begin{tabular}{|c|c|c|c|c|}
\hline & \multicolumn{3}{|c|}{ parámetros fenológicos } \\
\hline & & DB & NH & $\mathbf{A P}$ \\
\hline \multirow{3}{*}{ 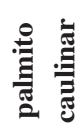 } & diámetro & $0,27 *$ & $0,42 * * *$ & $0,29 *$ \\
\hline & largo & ns & $0,40 * *$ & $0,53^{* * *}$ \\
\hline & peso & $0,23 *$ & $0,46^{* * *}$ & $0,46^{* * *}$ \\
\hline \multirow{3}{*}{ 产 } & diámetro & $0,54 * * *$ & $0,497 * * *$ & $0,51 * * *$ \\
\hline & largo & $0,34 *$ & $0,35^{*}$ & $0,36^{*}$ \\
\hline & peso & $0,59 * * *$ & $0,51 * * *$ & $0,53 * * *$ \\
\hline
\end{tabular}

$\mathrm{r}=$ coeficiente de correlación de Pearson; $\mathrm{ns}=(\mathrm{P}>0,05) ; *=(\mathrm{P}$ $<0,05) ; *=(\mathrm{P}<0,01) ; * * *=(\mathrm{P}<0,001)$.

como guía para definir el momento apropiado para la cosecha que han usado los productores de palmito en Brasil y en Costa Rica.

\section{DISCUSIÓN}

En una región como Guápiles, donde el déficit hídrico no es limitante, (como pudo confirmarse mediante mediciones del potencial hídrico al amanecer durante los períodos donde se presentó una leve disminución de las lluvias), la radiación solar aumenta su importancia como factor regulador del crecimiento y la productividad (Azam-Ali y Squire 2002). Evidentemente, los tratamientos experimentales evaluados se justifican en función del desarrollo del dosel y consecuentemente, de la cobertura del espacio y de la interceptación de la radiación incidente. La densidad de población, en términos no solamente de la cantidad de plantas por área sino también del arreglo espacial de las mismas generalmente se logra definir por prueba y error, aunque existe evidencia abundante de que el segundo aspecto no siempre ofrece los resultados esperados (Azam-Ali y Squire 2002). Es posible que en muchas especies tropicales, como la macadamia, los cítricos o el mango, los sistemas de siembra triangulares sean recomendables ya que la distancia entre árboles es grande y el dosel poblacional logra una mejor cobertura e interceptación de la radiación y con ello, un mejor control de las malezas, cuando la plantación es adulta. Una vista aérea de este arreglo consiste de una repetición de unidades de cuatro plantas con una en el centro, lo que no deja duda del mejor aprovechamiento espacial. En el caso del pejibaye, donde las plantas individuales producen rebrotes en forma inversamente proporcional a la densidad de población, como se observó en esta investigación, se obtendrá una compensación en el número de tallos cosechables por cepa y por unidad de área (Cuadro 7), que será más evidente en el largo plazo cuando la disposición de las cepas es triangular, por permitir un mejor aprovechamiento de la luz.

Los efectos de la competencia entre cepas resultan más notorios cuando la demanda por recursos aumenta en función de la mayor densidad de siembra. En esta investigación, el fertilizante, al ser aplicado por planta y no por área, compensó, de alguna forma la competencia entre plantas en una misma parcela. Por otra parte, el palmito propiamente no es un producto rico en nutrientes que demande una gran cantidad de energía por parte de la planta para su desarrollo, por lo que la mayor competencia posiblemente se observaría durante la producción de frutos, si la plantación fuese destinada para este propósito.

Normalmente, la producción de rebrotes es mayor cuando la densidad de la plantación es menor, debido a la mayor penetración de la radiación solar (Mora-Urpí et al. 1999). El número promedio de rebrotes aumentó a partir de la densidad de siembra de 3.333 plantas/ha hacia la densidad de 5.000 plantas/ha en función de la mayor cantidad de fertilizante químico o enmienda orgánica por ha (Cuadro 4), pero disminuyó en la mayor densidad, en función de la menor radiación fotosintéticamente activa (RFA) transmitida a la parte inferior del dosel. Resultado similar fue observado por Montenegro y Bogantes (2001), quienes observaron un menor número de rebrotes por cepa en plantaciones bajo árboles de laurel, donde la RFA era menor. La densidad de siembra, en el ámbito experimental evaluado en este trabajo, requiere de un análisis exhaustivo a un plazo de más duración, en donde debe incluirse otros parámetros adicionales, tal como la poda de exceso de rebrotes, para obtener una evaluación más completa sobre el manejo de la plantación para el propósito establecido. Este aspecto puede cobrar importancia en 
situaciones de escasez o de precios altos del almácigo, como suele ocurrir cuando se libera un nuevo cultivar para la siembra comercial.

Investigaciones realizadas por Clement (1995) y Neves et al. (2003) en condiciones climáticas diferentes a las nuestras, confirman los resultados obtenidos en esta investigación, particularmente el efecto de la densidad de siembra en la producción de palmito a los 16 mds.

Si bien el fertilizante orgánico no produjo efectos inmediatos o a corto plazo similares al fertilizante químico en el número y tamaño de los rebrotes, es importante considerar este aspecto desde una óptica de sostenibilidad. El fertilizante orgánico ofrece una excelente oportunidad comercial que por su importancia se discute en un artículo aparte (Chaimshon 2006a).

La lluvia es un factor detrimental para la permanencia del fertilizante en el suelo y es de esperar que el fertilizante inorgánico sea absorbido parcialmente después de su aplicación, mientras que una parte considerable es lavada por la escorrentía y la lixiviación. El fertilizante orgánico, aunque de mineralización gradual, no escapa a la fuerte lixiviación y su escasa presencia en la rizosfera podría ser mejor aprovechada por el sistema radical que por la parte aérea de la planta. Recordemos que aquella parte de la planta (raíz o tallo) más cercana al factor limitante, aprovecha mejor ese recurso (Russell 1977), ésto podría explicar en parte el marcado efecto del fertilizante orgánico tuviese un efecto tan obvio en el desarrollo radical (Chaimsohn 2006b). Este aspecto también sugiere la importancia de una evaluación a largo plazo, donde el efecto del fertilizante orgánico podría mostrar más claramente sus efectos beneficiosos.

En otras condiciones climáticas con precipitación pluvial similar (Bogantes 1999) o menor (De Oliveira et al. 2001; Ares et al. 2003; Nascimento et al. 2005) que la observada durante la conducción de este trabajo, la enmienda orgánica ha dado resultados promisorios en la producción de palmito.

\section{AGRADECIMIENTOS}

Los autores agradecen al CNPq-Brasil, por el otorgamiento de la beca de doctorado al primer autor, sin la cual este trabajo no se hubiera realizado. Al Centro de Investigación en Granos y Semillas de la Universidad de Costa Rica (UCR) por la indispensable ayuda financiera y logística. A la Vice Rectoría de Investigación de la UCR por el apoyo financiero. A Guillermo Solano, Carlos Arroyo y al personal de la UCR en la Estación Experimental Los Diamantes por el imprescindible apoyo en el trabajo de campo.

\section{LITERATURA CITADA}

ARES, A.; FALCAO, N.; YUYAMA, K.; YOST, R.S.; CLEMENT, C.R.. 2003. Response to fertilization and nutrient deficiency diagnostics in peach palm in Central Amazonia. Nutrient Cycling in Agroecosystems, 66: $221-232$.

ARROYO, C. 2004. Fenología e incidencia de plagas en cuatro variedades de pejibaye (Bactris gasipaes) para producción de palmito en Guápiles, Costa Rica. Tesis Mag. Sc., San José, C.R., Universidad de Costa Rica. 124 p.

AZAM-ALI, S.N.; SQUIRE, G.R. 2002. Principles of tropical agronomy. CAB International Publishing, Wallingford, UK. 236 p.

BOGANTES, A. 1999. Posibilidades de producción orgánica en palmito de pejibaye. In: Palmito de pejibaye (Bactris gasipaes Kunth): su cultivo e industrialización. San José, CR, Editorial de la Universidad de Costa Rica. p. 99 - 104.

BOGANTES, A. 2003. Evaluación de cuatro distancias de siembra y dos estrategias de control de malezas en pejibaye sin espinas (Bactris gasipaes K.) para palmito. Tesis Mag. Sc. San José, CR., Universidad de Costa Rica. 91 p.

BOVI, M.L.A.; GODOY JR, G.; TUCCI, M.L.S; SPIERING, S.H.. 2002. Nitrogen deficiency in peach palm (Bactris gasipaes kunth). Anales del XXVI International Horticultural Congress, ISHS, Toronto. p. 518.

CHAIMSOHN, F.P. 2000. Cultivo de pupunha e produção de palmito. Viçosa, Brasil, Ed. Aprenda Fácil. 121 p.

CHAIMSOHN, F.P. 2006a. Análisis económico-financiero de la producción de palmito para consumo al natural en función de la población de plantas y del tipo de 
fertilización. In: Producción y calidad del palmito al natural, en función de la población, del arreglo de plantas y del tipo de fertilización. Tesis Doctorado San José, C.R., Universidad de Costa Rica. p. 151 - 172.

CHAIMSOHN, F.P. 2006b. La fertilización orgánica favorece el desarrollo radicular en pejibaye (Bactris gasipaes). In: Producción y calidad del palmito al natural, en función de la población, del arreglo de plantas y del tipo de fertilización. Tesis Doctorado San José, C.R., Universidad de Costa Rica. p. 139 - 150.

CLEMENT, C.R. 1995. Growth and genetic analysis of pejibaye (Bactris gasipaes Kunth, Palmae) in Hawaii. Ph.D. Dissertation, Honolulu, University of Hawaii at Manoa, xvi + 221 p.

CLEMENT, C.R.; BOVI, M.L.A.. 1999. Padronização de medidas de crescimento e produção em experimentos com pupunheiras para palmito. Acta Amazônica, 30(3): $349-362$.

CLEMENT, C.R.; MANSHARDT, R.M.; DEFRANK, J.; CATHERINE, G.; NAGAI, N. 1996. Introduction of pejibaye for heart-of-palm in Hawaii. Hort. Science, 31(5): $765-768$.

DE OLIVEIRA, A.P.; ALVES, A.U.; CANDEIA, B.L.; BENVINDA, J.M.S.; BARBOSA, L.J.N.; SILVA, J.V. 2001. Desenvolvimento da pupunheira cultivada com fertilizante orgânico e mineral. Horticultura Brasileira, CD-ROM, 2001. 19 (Suplemento).

MOLINA R., E. A. 2000. Manual de suelos y nutrición de pejibaye para palmito. San José, C.R., Asociación Costarricense de Ciencia del Suelo. 42 p.

MONTENEGRO, J.; BOGANTES, A.. 2001. Palmito de pejibaye (Bactris gasipaes) cultivado bajo diferentes densidades de laurel (Cordia alliodora). Agronomía Costarricense, 25(2): 73 - 79.

MORA-URPÍ, J.; BOGANTES, A.; ARROYO, C.; RIVERA, C.L.. 1999. Densidades de siembra. In: Mora Urpí, J. y Gainza E., J. eds. Palmito de pejibaye (Bactris gasipaes Kunth): su cultivo e industrialización. San José, C.R., Editorial de la Universidad de Costa Rica. p. 107 - 113.

NASCIMENTO, J.T.; OLIVEIRA, A.P.; SOUZA, A.P.; SILVA, I.F.; ALVES, A.U. 2005. Rendimento de palmito de pupunheira em função da aplicação de esterco bovino e adubação química. Horticultura Brasileira 23 (1): 19-21.

NEVES, E.J.M.; SANTOS, A.F.; MARTINS, E.G.; AHRENS, S.; KALIL, A.N. 2003. Efeito de diferentes espaçamentos na produção de pupunha para palmito no Litoral do Paraná $-1^{\circ}$ corte. Boletim da Pesquisa Florestal, 46: 69 - 81.

PEREIRA Q., J. DE D. 2002. Perfil físico-químico del palmito de pejibaye (Bactris gasipaes Kunth) producido en Costa Rica. Proyecto de Graduación de Licenciatura en Tecnología de Alimentos. San José, C.R., Universidad de Costa Rica. 66 p..

PROMOTORA DEL COMERCIO EXTERIOR DE COSTA RICA (PROCOMER). 2006. Costa Rica: Estadísticas de exportación 2005. San José, PROCOMER, 231 p.

RAMOS, A. 2002. Análise do desenvolvimento vegetativo e produtividade da palmeira pupunha (Bactris gasipaes Kunth) sob níveis de irrigação e adubação nitrogenada. Tesis Doctorado. Piracicaba, Brasil, Escola Superior de Agricultura "Luis de Queiroz". 113 p.

RUSSELL, R.S. 1977. Plant Root Systems. Their function and interaction with the soil. McGraw-Hill Book Co., London, UK.

UNITED STATES DEPARTMENT OF AGRICULTURE (USDA) Y NATURAL RESOURCES CONSERVATION SERVICE (NRCS). 2006. Keys to Soil Taxonomy. USDA y NRCS, 341 p. 10 ed. Consultado en 08 de junio de 2006. Disponible en: http://soils.usda. gov/technical/classification/tax_keys/keys.pdf

VANDEVIVERE, P.; RAMÍREZ, C. 1995. Bioensayo microbiano para determinar los nutrimentos disponibles en abonos orgánicos. Boltec, 28(2): 90 - 96.

VEGA, F.V.A.; BOVI, M.L.A.; BERTON, R.S.; GODOY JR, G. 2004. Aplicação de biosólido na implantação da cultura da pupunheira. Horticultura Brasileira 22 (1): 131 - 135.

VIILLALOBOS, E.; UMAÑA, C.H.; CHINCHILLA, C. 1992. Etat d`hydratation du palmier a huile, en réponse a la sécherese au Costa Rica. Oléagineux, 47:1-7.

YUYAMA, K., W.B. CHÁVEZ F., B.G. PEREIRA, I.A. SILVA. 2005. Efeito da densidade de plantas e da adubação NPK na produção inicial de palmito de pupunheira. Revista Brasileira de Ciência do Solo, 29: 373 - 378. 